\title{
Wide-field parallel mapping of local spectral and topographic information with white light interference microscopy
}

\author{
Sébastien Marbach,,${ }^{1,}{ }^{*}$ Rémy Claveau, ${ }^{1}$ Fangting Wang, ${ }^{1}$ Jesse Schiffler, ${ }^{1}$ \\ Paul Montgomery, ${ }^{1}$ and Manuel Flury ${ }^{1}$ \\ ${ }^{1}$ ICube, Université de Strasbourg, CNRS, INSA, F-67000 Strasbourg, France \\ *Corresponding author: marbachs@unistra.fr
}

Received XX Month XXXX; revised XX Month, XXXX; accepted XX Month XXXX; posted XX Month XXXX (Doc. ID XXXXX); published XX Month XXXX

Fourier analysis of interferograms captured in white light interference microscopy is proposed for performing simultaneous local spectral and topographic measurements at high spatial resolution over a large field of view. The technique provides a wealth of key information on local sample properties. We describe the necessary processing and calibration involved to produce reflectivity maps of spatially extended samples. This enables precise and fast identification between different materials at a local scale of $1 \mu \mathrm{m}$. We also show that the recovered spectral information can be further used for improving topography measurements, particularly in the case of samples combining dielectric and conducting materials in which the complex refractive index can result in nanometric height errors. (C) 2020 Optical Society of America

\footnotetext{
Optical spectrometers and ellipsometers are the main systems used to measure the spectroscopic information of materials. The former uses the principles of dispersion or Fourier-Transform spectroscopy, while the latter are based on the measurement of the change in polarization. The spectral information obtained is averaged over the illumination spot size of the instruments, which typically covers several $\mathrm{mm}^{2}$ for standard spectrometers [1], down to thousands of $\mu \mathrm{m}^{2}$ for ellipsometers. These systems cannot therefore provide spectral measurement of materials at the micrometer scale, nor perform imaging over microscopic areas. For samples with micrometric dimensions, new methods for measuring spectral information with a very high spatial resolution have been developed, such as s-SNOM (Spectral Scanning Near-field Optical Microscopy) [2] and hyperspectral imaging microscopy [3,4]. Nevertheless, as the former is a pointwise measurement technique, it requires long scanning times to image a large area of the sample, while the latter requires scanning in either the spatial or the spectral domains.

Fast growth in new nanotechnologies and more advanced studies of living matter at the micrometer/nanometer scale have generated increasing demands in the high resolution and speed of local characterizations [2-4]. To meet these requirements, White Light Interference Microscopy (WLIM) has become a standard tool for surface roughness and 3D structural measurement at the microscopic scale. The full-field imaging capability [5] enables measurements of surface topography and roughness with both high lateral resolution (about $400 \mathrm{~nm}$ ) and axial sensitivity (a few $\mathrm{nm}$ ) over relatively large
}

Fields of View (FoV). Fast processing techniques such as Phase Shifting Microscopy (PSM) and Coherence Scanning Interferometry (CSI) [5-7] are typically used to recover topographic information. The addition of a color camera to such a system enables material identification to a certain extent [8] but because it is not quantitative, would not be able to distinguish between two similar looking or metamer colored materials [9]. On the other hand, by using an approach based on performing a Fourier Transform of the fringe signal, quantitative spectral information can be obtained locally [10-12]. This information can also be resolved over depth under certain illumination conditions if the sample is transparent [12]. This method has also been successfully applied for measuring the reflectivity of thin and thick films in order to deduce their local thickness and dispersion law $[13,14]$.

In this paper we present how WLIM can provide simultaneous 3D spatial and spectral 2D maps of materials with micrometer lateral resolution by using a single axial scan. Signal processing similar to that used in classical Fourier-Transform spectroscopy is used but without the need for an additional spectrometer. Given the full-field imaging ability of WLIM, this method allows fast acquisition of the spectral information over a large area, whilst preserving the capacity for distinguishing spectrally different materials with micrometric dimensions.

CSI consists in acquiring the interferometric signal over depth (Z) within a large area (XY) of the sample measured as illustrated in Fig. 1(a). The signal is extracted over depth pixel by pixel (Fig. 1(b)) and then processed to obtain information about the sample reflectivity in 
different Regions Of Interest (ROI). Since white light is used, the intensity of the interferometric signal $I$ corresponds to the sum of the interference signals at each wavelength $\lambda$, as given by Eq. (1):

$$
\begin{aligned}
& I(z)=\int_{\omega_{1}}^{\omega_{2}} \frac{S_{\text {sys }}(\omega)}{4}\left(R_{\text {ref }}(\omega)+R_{\text {sam }}(\omega)\right)+ \\
& \frac{S_{\text {sys }}(\omega)}{2} \sqrt{R_{\text {ref }}(\omega)} \sqrt{R_{\text {sam }}(\omega)} \cos (\Delta \varphi(\omega, z)+\Phi) \mathrm{d} \omega
\end{aligned}
$$

where $\omega$ is the frequency and $\omega_{1}$ and $\omega_{2}$ are the bandwidth limits of the source. $S_{\text {sys }}$ is the spectral response of the whole system, given by the product of the source spectrum, the spectral response of the camera and the spectral transmittance of the entire optical system. $R_{r e f}$ and $R_{\text {sam }}$ are the reflectivity of the reference mirror and the sample analyzed respectively. $\Delta \varphi$ is the phase difference between the reference and the object arms, and $\Phi$ is the phase on reflection error.

The processing then consists in removing the offset in Eq. (1) and conserving only the part modulated by the cosine function. For spectral analysis, the optical path difference $\delta$ and the wavenumber $\sigma$, defined as the inverse of the wavelength, are used. The limits of the integral are extended to infinity (with the spectral response equal to 0 outside the boundaries $\omega_{1}$ and $\omega_{2}$ ). Finally, a Fourier Transform (FT) is applied, and its modulus gives the spectral response of the system multiplied by the sample and reference mirror reflectivity, as presented in Eq. (2). This result is known as the Non-Calibrated Spectral response (NCS).

$$
|F T[I(\delta)]|=\frac{1}{4} S_{s y s}(\sigma) \sqrt{R_{r e f}(\sigma)} \sqrt{R_{\text {sam }}(\sigma)}=\operatorname{NCS}(\sigma)
$$

To determine the sample reflectivity, $S_{s y s}$ and $R_{r e f}$ are estimated beforehand through a calibration step. This consists in measuring the $N C S$ of a calibration sample (a silicon wafer in our case) having a spectral reflectivity previously measured using a classic optical spectrometer. According to Eq. (2), the value of $S_{\text {sys }}(\sigma) \cdot\left[R_{\text {ref }}(\sigma)\right]^{1 / 2}$ can then be determined, which is independent of the sample and specific to the system. This product is the Spectral Transfer Function (STF). Finally, by keeping the same parameters (i.e. source intensity and optical arrangement) for the measurement step as in the calibration step, the reflectivity of any sample can be determined quantitatively pixel by pixel with the formula given in Eq. (3):

$$
R_{\text {sam }}(\sigma)=\left|\frac{N C S(\sigma)}{\operatorname{STF}(\sigma)}\right|^{2}
$$

The system used to conduct the experiments is composed of a white light interferometer in a Linnik configuration mounted on a breadboard [15] as illustrated in Fig.1(c). The effects of environmental noise are reduced by placing the whole system in a special temperaturecontrolled anti-vibration room mounted on air cushions. The light emitted by the white LED (Thorlabs MBB1L3) passes through the Köhler illuminator and is then divided into two beams by the beamsplitter. Both the reference and object beams travel through identical long-working distance Mitutoyo objectives (M Plan Apo 20x/0.42, infinity corrected), before being reflected by a reference mirror (RM) and the sample. These reflected beams are recombined to produce a fringe pattern superimposed on the sample image, which is captured using a 16-bit cooled camera (PCO Edge 4.2 USB 3.0). The sample is placed on a position controlled piezo stage (PZT PI P621-ZCD) to allow scanning along the optical axis with nanometer sensitivity. The setup is controlled by homemade software developed in LabVIEW. The setup parameters between the calibration and the measurement steps stay the same. To avoid any spectrum distortion caused by large illumination apertures [16], the aperture diaphragm (AD) is closed during the image

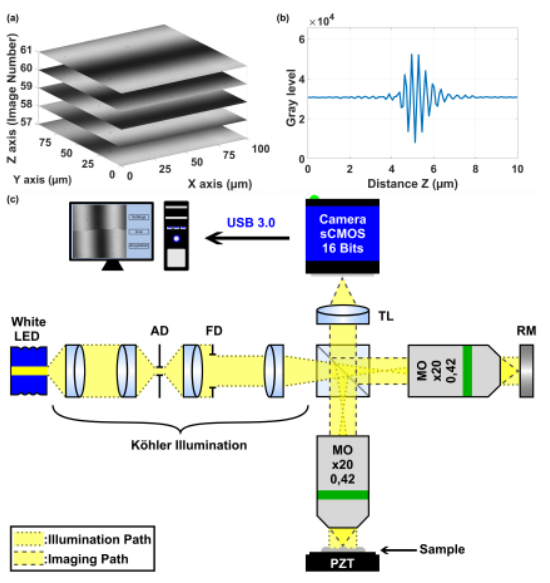

Fig. 1. (a) XYZ stack of interferometric images. (b) Interferometric signal extracted at one pixel. (c) Layout of the white light interferometer used to perform the experiments. The aperture diaphragm (AD) controls the illumination angle cone and the field diaphragm (FD) the field of view. The Tube Lens (TL) produces the image on the camera.

capture. A 3x3 pixel averaging filter is applied to each interferometric image to reduce camera-related noise (readout and dark current) before processing. Due to the larger size of the Airy diffraction disk (about $1 \mu \mathrm{m}$ ) compared to the $3 \times 3$ pixel area, no information is lost in terms of lateral resolution. The interferometric stack is acquired over a depth of $10 \mu \mathrm{m}$, with a piezoelectric displacement step of $\lambda / 8$. With an effective wavelength of $660 \mathrm{~nm}$ being used in this setup, the piezo step is equal to $82.5 \mathrm{~nm}$. Each interferometric image is obtained using temporal averaging of 10 images. The packet of interference fringes is windowed with a $9 \mu \mathrm{m}$ wide Hamming apodization window. A zeropadding operation followed by interpolation enables the reflectivity to be then displayed with a step of $1 \mathrm{~nm}$, although this does not correspond to the spectral resolution. This latter value depends mostly on the apodization window width, the wider the interferogram, the better being the spectral resolution. The use of a $9 \mu \mathrm{m}$ wide Hamming window leads to an estimated spectral resolution of about $25 \mathrm{~nm}$ at $\lambda=500 \mathrm{~nm}$ and $44 \mathrm{~nm}$ at $\lambda=660 \mathrm{~nm}$ according to the FT sampling. As suggested in [17], averaging of the reflectivity is performed over 6 processed interferometric stacks to obtain more accurate spectral information. This allows a good trade-off to be maintained between accuracy and acquisition time ( $\sim 5 \mathrm{~min})$. The repeatability of the standard deviation (Std) to the averaged reflectivity ratio, $\mathrm{Std}(\mathrm{R}) / \mathrm{R}$, was measured to be 0.02 when making 25 identical successive measurements over 3 hours. The spectral results hereafter are displayed with their spatial Std, which gives an idea of the minimum reflectivity difference that would be identifiable. Processing of the whole field of view (401x404 pixels ${ }^{2}$ $=130 \times 131 \mu \mathrm{m}^{2}$ ) is performed using MATLAB to take advantage of the matrix computing power and only requires a little less than 2 min on a PC equipped with an Intel i7-960 processor and 24 Go RAM.

The validity of the proposed method was first demonstrated by identifying two different materials through their spectral properties. A thin gold layer $(\sim 50 \mathrm{~nm})$ was deposited onto an aluminum substrate. As illustrated in Fig. 2(a), the image captured with the camera does not allow an easy identification of which part of the sample is composed of aluminum or gold, as the gray level intensity measured by the camera is similar for both materials. With the method presented above, it is possible to extract multiple reflectivity maps, three of which are displayed in Fig. 2(b), (c) and (d) for the wavelengths $500 \mathrm{~nm}, 575 \mathrm{~nm}$ and $750 \mathrm{~nm}$. This provides a clear distinction between the materials based on their reflectivity at the chosen wavelength. Although the $575 \mathrm{~nm}$ reflectivity map presents an almost similar spectral response for each material (about 90\%), the $500 \mathrm{~nm}$ map, where the lower and 

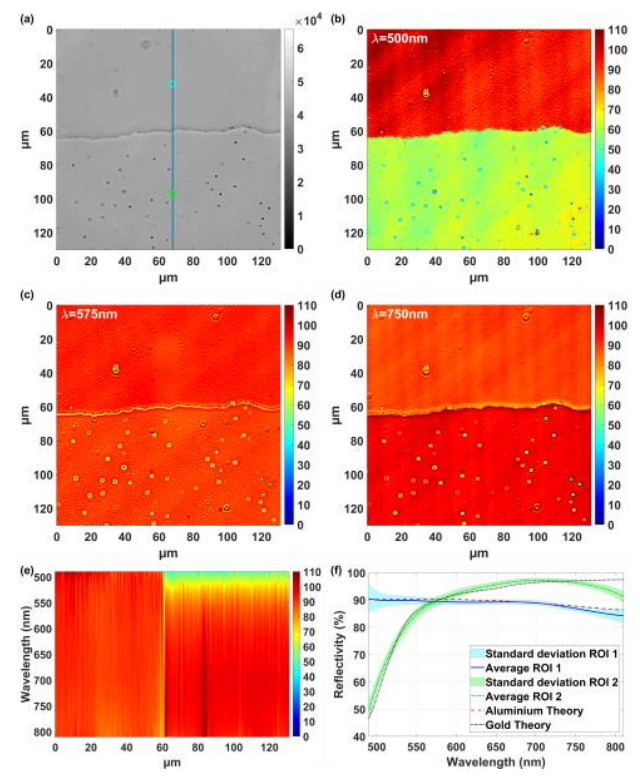

Fig. 2. (a) Gray-level image of gold on aluminum sample. Reflectivity map at a wavelength of $500 \mathrm{~nm}$ (b), $575 \mathrm{~nm}$ (c), $750 \mathrm{~nm}$ (d) and with $1 \mathrm{~nm}$ steps from $500 \mathrm{~nm}$ to $800 \mathrm{~nm}$ in Visualization 1. (e) Spectral cross section along the blue line in (a). (f) Reflectivity as a function of the wavelength from the cyan square (ROI 1) and green square (ROI 2) in (a) compared to theoretical data: red dashed line for aluminum and black dotted line for gold.

upper parts have a reflectivity of about $55 \%$ and $90 \%$ respectively is particularly relevant for identifying the presence of the two different materials. It should be noted that at this wavelength the influence of the noise is more significant, which is explained by the LED emission intensity that substantially decreases below $520 \mathrm{~nm}$ and above $775 \mathrm{~nm}$. This leads to a STF closer to 0 at these wavelengths and thus to a greater measurement uncertainty, as shown with increasing Std values Fig. 2(f). In Visualization 1, the reflectivity map is displayed from $500 \mathrm{~nm}$ to $800 \mathrm{~nm}$ in steps of $1 \mathrm{~nm}$. The spectral cross-section in Fig. 2(e), taken along the blue line in Fig. 2(a), also clearly shows the heterogeneity of the sample composition. The plot in Fig. 2(f) is obtained by analyzing the average and standard deviation reflectivities measured in a $3.25 \times 3.25 \mu^{2}$ area represented in cyan (labelled ROI 1) in the upper part and in green (labelled ROI 2) in the lower part in Fig. 2(a). Theoretical reflectivities for aluminum and gold [18] are represented by the red dashed line and black dotted line in Fig. 2(f). The shape of the reflectivity spectra together with the reflectivity maps make it possible to deduce that the upper material is aluminum and the lower one is gold.

The spectral characterization of the samples presented above only uses the magnitude of the FT to determine the reflectivity. The angle $\Delta \varphi$ given by the phase of the FT provides different information and can be used to extract the sample topography $h$, as shown by Eq. (4):

$$
h(x, y)=\frac{1}{4 \pi} \frac{d(\Delta \varphi)}{d \sigma} \text { with } \Delta \varphi(\sigma)=\arg \{F T[I(x, y, \delta)]\}
$$

This is similar to that used in Fourier Domain Analysis [19], which combines the advantages of CSI and PSM, that is, an axial sensitivity of a few $n m$ with the removal of the $\lambda / 2$ steps uncertainty. Consequently, all the information contained in the FT of the interferogram is used, resulting in simultaneous mapping of spectral reflectivity and topographic information. These are obtained from the same captured image data. The result provided by the Eq. (4) is used to command the unwrapping of the topography obtained from the phase value at a wavelength of $\lambda=671 \mathrm{~nm}$ for which the signal to noise ratio is optimal.

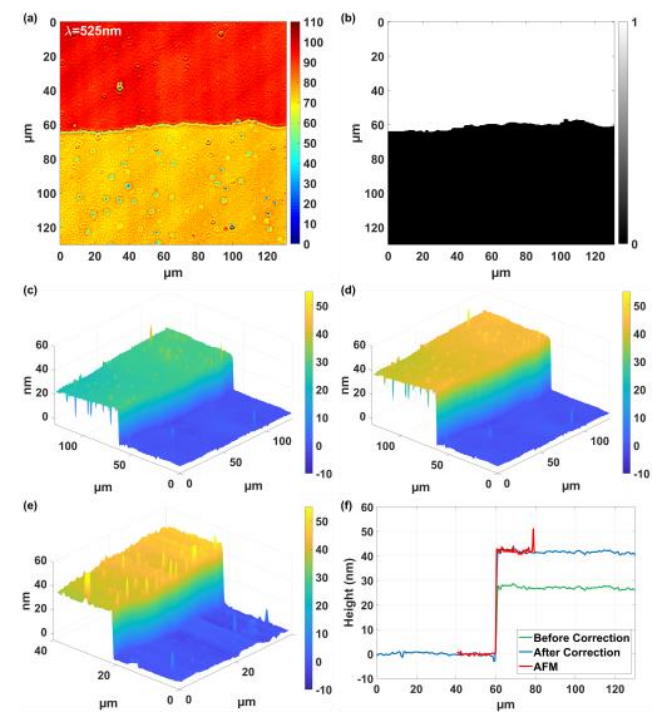

Fig. 3. (a) Reflectivity map at $\lambda=525 \mathrm{~nm}$ for a sample made of gold deposited on an aluminum substrate. (b) Binary map extracted from (a) after image processing. Topography measured with the proposed method before (c) and after (d) reflection phase shift difference correction. (e) Topography measured with AFM. (f) Profile extracted from each topographic map in centerline in green, blue and red respectively for CSI without and with correction and AFM.

As the sample measured is composed of two distinct metals, different phase shifts are introduced on reflection [20]. At this wavelength, the phase shift reflection for unpolarized light is equal to $\sim 1.314$ rad for gold [21] and $1.450 \mathrm{rad}$ for aluminum [22], leading to a phase shift difference of $0.137 \mathrm{rad}$ or a height difference of $14.6 \mathrm{~nm}$. To correct the error, the material location needs to be identified. This is easily achieved using the parallel measurement of the spectral response. For instance, the spectral map is extracted at $\lambda=525 \mathrm{~nm}$, as shown in Fig. 3(a). At this wavelength, the reflectivity of the gold is about $75 \%$ compared to about $90 \%$ for the aluminum. The binary map in Fig. 3(b) is obtained via Otsu's binarization algorithm of the reflectivity map in (a) followed by a $3 \times 3$ median filter and successive morphological closing and opening with a 3-pixels radius structuring element to remove the points missed in the binarization process. The aluminum and gold are defined by values of 1 and 0 . Now that the materials are computationally identified, the height correction between the aluminum and gold can be applied. The topographies before and after correction are illustrated in Fig. 3(c) and (d). They are compared to the topography obtained in Fig. 3(e) using an AFM Park Systems XE-70 and used as a gold-standard reference measurement (z-resolution about $1 \mathrm{~nm}$ in air in the noncontact mode). A profile in the center of each topographic view is plotted in Fig. 3(f) for comparison. After correction, the proposed technique provides a height measurement that matches very well with that obtained from the AFM system. Indeed, the average height is equal to $42 \pm 0.5 \mathrm{~nm}$ after correction and $41 \pm 3 \mathrm{~nm}$ with the AFM. The small difference can be explained by the system noise, the measurement location which can be slightly different between each system and the phase shift value chosen [21,22]. The error is only $2.22 \%$ and below $1 \mathrm{~nm}$. As a comparison an error of $33.35 \%$ would have been obtained without the correction method proposed.

Finally, local reflectivity measurements were performed on a microstructured element. The sample is a negative 1951-USAF resolution target standard (Model Edmund Optics \#38-256) made of a deposition of chromium on a glass substrate. The element 1 of the group 6 was imaged in Fig. 4(a). Fig. 4(b) shows the $660 \mathrm{~nm}$ reflectivity map. The $8 \mu \mathrm{m}$ glass lines are clearly contrasted with a reflectivity of about $4 \%$ 

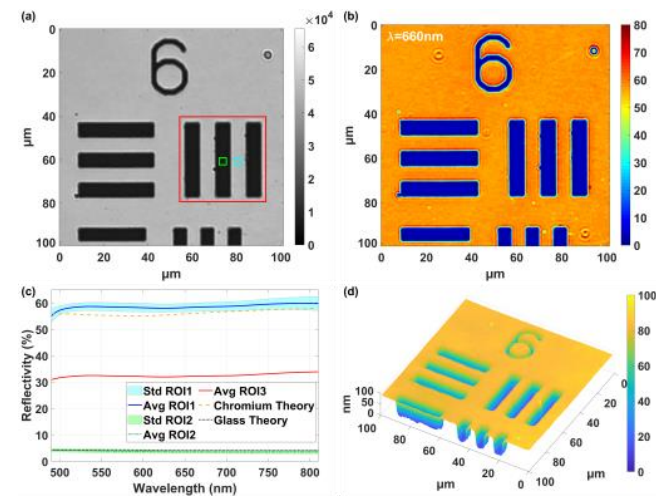

Fig. 4. (a) Gray-level image of the negative 1951-USAF resolution target. (b) Reflectivity map at a wavelength of $660 \mathrm{~nm}$, and with $1 \mathrm{~nm}$ steps from $500 \mathrm{~nm}$ to $800 \mathrm{~nm}$ in Visualization 2. (c) Reflectivity as a function of the wavelength from the cyan square (ROI 1), green square (ROI 2) and red square (ROI 3) in (a) compared to theoretical data: yellow dashed line for chromium and black dotted line for glass. (d) Topography measured with interferometer after phase on reflection difference correction.

compared to the chromium whose reflectivity is about 58\%. Fig. 4(c) gives the average and standard deviation of several reflectivity spectra obtained by analyzing each pixel inside the $3.25 \times 3.25 \mu \mathrm{m}^{2}$ cyan and green areas (labelled as ROI 1 and 2) plotted in Fig. 4(a). Theoretical reflectivities for chromium and glass are plotted in yellow dashed and black dotted lines in Fig. 4(c) for comparison, showing a very good match between the theoretical and measured spectra. While the shapes of the simulated and measured chromium reflectivity spectra are identical, there is a $2.5 \%$ offset difference between them. This can be explained by the properties of the chromium used in the simulation that might slightly vary from the material used in the experiment. A variation in the light intensity between the calibration and measurement steps could also provide another explanation. The quasi-constant reflectivity of both the chromium and glass in the visible spectral range is well represented in Fig. 4(c) and also in the Visualization 2 presenting the 2D reflectivity map evolution from $500 \mathrm{~nm}$ to $800 \mathrm{~nm}$ in steps of $1 \mathrm{~nm}$. These results illustrate the ability of the method to distinguish the nature of the material from $\mu$ m-sized elements. It also highlights its benefits compared to classical spectral measurement devices. Indeed, the reflectivity measured with a standard spectrometer would have been at best similar to the reflectivity spatially averaged (shown in Fig. 4(c)) over the $40 \times 40 \mu \mathrm{m}^{2}$ red square plotted in Fig. 4(a) (labelled ROI 3). In this case the information from both materials is mixed together preventing any spatially-resolved analysis and further sample identification. As explained previously, the topography can also be obtained by analyzing the phase of the same data set acquired. Light reflection on a chromium surface leads to an error in the measured height of $14.5 \mathrm{~nm}$ at $\lambda=671 \mathrm{~nm}$ [23], which can be corrected by applying the method detailed previously. The chromium position is identified via spectral characterization and its topography subsequently corrected (Fig. 4(d)). The comparison with an AFM measurement across the vertical glass line gives an average height of $76.5 \pm 1.5 \mathrm{~nm}$. With the proposed method an average height of $70.5 \pm 1 \mathrm{~nm}$ is obtained after correction. The possible source of errors is identical to those discussed previously and can explain the difference of $6 \mathrm{~nm}$. To-date, the method proposed has only been tested on flat plateau-like structures. In the case of samples presenting high local slope or curvature, it would be expected that the effective spectrum be modified, as discussed in [24], requiring further investigation and a strategy for correction.

In conclusion, the ability to measure spectral reflectivity in a full-field configuration with locally resolved values has been demonstrated experimentally using a white light interferometer. Clear identification of samples composed of two different materials has been performed at the micrometer scale. From the same interferometric stack, topography was obtained in addition to the spectral information and further corrected using known values of the phase on reflection error introduced at the reflection. This paves the way for fast characterization of spatially extended samples with micro-structured elements that require both a large FoV and high spatial resolution. In this letter, $\mathrm{x} 20$ objectives were used to rapidly image an area of $130 \mathrm{x} 131 \mu^{2}$ with an acquisition time $<5 \mathrm{~min}$ and a processing time $<2 \mathrm{~min}$. The use of objectives with lower magnification would lead to characterizing even larger FoV while preserving the same acquisition time, but at the cost of a decreased lateral resolution. Since the acquisition and processing times are only software/hardware limited, it is expected that further work will allow significant reductions, particularly for the acquisition time so that the technique could be used with classical interferometers on standard optical tables without compromising the measurement sensitivities.

Acknowledgments and Funding. We would like to thank Stéphane Roques of the $\mathrm{C}^{3} \mathrm{FAB}$ platform at ICube for the preparation of the samples and Strasbourg University for financial support.

Disclosures. The authors declare no conflicts of interest.

\section{References}

1. S. P. Davis, M. C. Abrams and J. W. Brault, Fourier Transform Spectrometry (Elsevier, 2001).

2. I. Amenabar, S. Poly, W. Nuansing, E. H. Hubrich, A. A. Govyadinov, F. Huth, R. Krutokhvostov, L. Zhang, M. Knez, J. Heberle, A. M. Bitnner and R. Hillenbrand, Nat Commun. 4, 2890 (2013).

3. S. J. Leavesley, N. Annamdevula, J. Boni, S. Stocker, K. Grant, B. Troyanovsky, T. C. Rich and D. F. Alvarez, J. Biophotonics 5, 67 (2012).

4. R. D. Alcock and J. M. Coupland, Meas. Sci. Technol. 17, 2861 (2006).

5. P. de Groot, Adv. Opt. Photon. 7, 1 (2015).

6. P. Lehmann, S. Tereschenko and W. Xie, Surf. Topogr.: Metrol. Prop. 4, 024004 (2016).

7. P. C. Montgomery, F. Anstotz, J. Montagna, D. Montaner, L. Pramatarova and E. Pecheva, J. Phys.: Conf. Ser. 253, 012017 (2010).

8. J. L. Beverage, X. Colonna de Lega, and M. F. Fay, Proc. SPIE 9203 (2014).

9. A. Akbarinia and K. R. Gegenfurtner, J. Opt. Soc. Am. A 35, B231 (2018).

10. A. Dubois, J. Moreau and C. Boccara, Opt. Express 16, 17082 (2008).

11. G. Latour, J. Moreau, M. Elias and J. M. Frigerio, Opt. Comm. 283, 4810 (2010).

12. R. Claveau, P. C. Montgomery, M. Flury and D. Montaner, Opt. Express 25, 20216 (2017).

13. R. Claveau, P. C. Montgomery, M. Flury and G. Ferblantier, J. Opt. Mat. 86, 100 (2018).

14. T. Guo, Z. Chen, M. Li, J. Wu, W. Fu and X. Hu, Appl. Opt. 57, 2955 (2018).

15. S. Marbach, C. Cordier, R. Claveau, T. Engel, P. C. Montgomery and M. Flury, Proc. SPIE 11352 (2020).

16. A. Morin and J. M. Frigerio, Appl. Opt. 51, 3431 (2012).

17. R. Claveau, P. C. Montgomery, M. Flury and D. Montaner, Proc. SPIE 9890 (2016).

18. F. Canova, O. Uteza, J. P. Chambaret, M. Flury, S. Tonchev, R. Fechner and O. Parriaux, Opt. Express 15, 15324 (2007).

19. P. De Groot and L. Deck, Opt. Lett. 18, 1462 (1993).

20. A. Dubois, Appl. Opt. 43, 1503 (2004).

21. P. B. Johnson and R. W. Christy, Phys. Rev. B. 6, 4370 (1972).

22. A. D. Rakić, Appl. Opt. 34, 4755 (1995).

23. P. B. Johnson and R. W. Christy, Phys. Rev. B. 9, 5056 (1974).

24. P. Lehmann, S. Tereschenko, B. Allendorf, S. Hagemeier, and L. Hüser, J. Eur. Opt. Soc.-Rapid Publ. 15, 5 (2019). 
1. S. P. Davis, M. C. Abrams, and J. W. Brault, "Why chose a fourier transform spectrometer?," in Fourier Transform Spectrometry (Elsevier, 2001), pp. 17-27.

2. I. Amenabar, S. Poly, W. Nuansing, E. H. Hubrich, A. A. Govyadinov, F. Huth, R. Krutokhvostov, L. Zhang, M. Knez, J. Heberle, A. M. Bittner, and R. Hillenbrand, "Structural analysis and mapping of individual protein complexes by infrared nanospectroscopy," Nature Communications 4, (2013).

3. S. J. Leavesley, N. Annamdevula, J. Boni, S. Stocker, K. Grant, B. Troyanovsky, T. C. Rich, and D. F. Alvarez, "Hyperspectral imaging microscopy for identification and quantitative analysis of fluorescently-labeled cells in highly autofluorescent tissue," Journal of Biophotonics 5, 67-84 (2012).

4. R. D. Alcock and J. M. Coupland, "A compact, high numerical aperture imaging Fourier transform spectrometer and its application," Meas. Sci. Technol. 17, 2861-2868 (2006).

5. P. de Groot, "Principles of interference microscopy for the measurement of surface topography," Advances in Optics and Photonics 7, 1 (2015).

6. P. Lehmann, S. Tereschenko, and W. Xie, "Fundamental aspects of resolution and precision in vertical scanning white-light interferometry," Surface Topography: Metrology and Properties 4, 024004 (2016).

7. P. C. Montgomery, F. Anstotz, J. Montagna, D. Montaner, L. Pramatarova, and E. Pecheva, "Towards real time 3D quantitative characterisation of in situ layer growth using white light interference microscopy," Journal of Physics: Conference Series 253, 012017 (2010)

8. J. L. Beverage, X. C. de Lega, and M. F. Fay, "Interferometric microscope with true color imaging," Proc. SPIE 9203, 92030S (2014).

9. A. Akbarinia and K. R. Gegenfurtner, "Color metamerism and the structure of illuminant space," J. Opt. Soc. Am. A 35, B231-B238 (2018).

10. A. Dubois, J. Moreau, and C. Boccara, "Spectroscopic ultrahighresolution full-field optical coherence microscopy," Optics Express 16, 17082 (2008).

11. G. Latour, J. Moreau, M. Elias, and J.-M. Frigerio, "Microspectrometry in the visible range with full-field optical coherence tomography for single absorbing layers," Optics Communications 283, 4810-4815 (2010).

12. R. Claveau, P. Montgomery, M. Flury, and D. Montaner, "Depthresolved local reflectance spectra measurements in full-field optical coherence tomography," Optics Express 25, 20216 (2017).

13. R. Claveau, P. Montgomery, M. Flury, and G. Ferblantier, "Local inspection of refractive index and thickness of thick transparent layers using spectral reflectance measurements in low coherence scanning interferometry," Optical Materials 86, 100-105 (2018).

14. T. Guo, Z. Chen, M. Li, J. Wu, X. Fu, and X. Hu, "Film thickness measurement based on nonlinear phase analysis using a Linnik microscopic white-light spectral interferometer," Applied Optics 57, 2955 (2018).

15. S. Marbach, C. Cordier, R. Claveau, T. Engel, P. C. Montgomery, and M. Flury, "White light interference microscopy system design," Proc. SPIE 11352, 113521B (2020).

16. A. Morin and J.-M. Frigerio, "Aperture effect correction in spectroscopic full-field optical coherence tomography," Applied Optics 51, 3431 (2012).

17. R. Claveau, P. C. Montgomery, M. Flury, and D. Montaner, "Local reflectance spectra measurements of surfaces using coherence scanning interferometry," Proc. SPIE 9890, 98900Q (2016).

18. F. Canova, R. Clady, J.-P. Chambaret, M. Flury, S. Tonchev, R. Fechner, and O. Parriaux, "High-efficiency, broad band, highdamage threshold high-index gratings for femtosecond pulse compression," Optics Express 15, 15324 (2007).
19. P. de Groot and L. Deck, "Three-dimensional imaging by subNyquist sampling of white-light interferograms," Optics Letters $\mathbf{1 8}$ 1462 (1993)

20. A. Dubois, "Effects of phase change on reflection in phasemeasuring interference microscopy," Applied Optics 43, 1503 (2004).

21. P. B. Johnson and R. W. Christy, "Optical Constants of the Noble Metals," Physical Review B 6, 4370-4379 (1972).

22. A. D. Rakić, "Algorithm for the determination of intrinsic optical constants of metal films: application to aluminum," Applied Optics 34, 4755 (1995).

23. P. B. Johnson and R. W. Christy, "Optical constants of transition metals: Ti, V, Cr, Mn, Fe, Co, Ni, and Pd," Physical Review B 9, 5056-5070 (1974).

24. P. Lehmann, S. Tereschenko, B. Allendorf, S. Hagemeier, and L. Hüser, "Spectral Composition of Low-Coherence Interferograms at High Numerical Apertures," Journal of the European Optical Society-Rapid Publications 15, 5 (2019). 